\title{
fMRI of human visual cortex
}

SIR - The primate visual system contains many distinct visual areas, each with its own rich structure'. Few detailed measurements of these areas have been made in humans, however, because of the poor spatial resolution of functional neuroimaging methods. For example, the spatial resolution in positron emission tomography (PET) studies $^{2}$ is typically $7-10 \mathrm{~mm}$. Using functional magnetic resonance imaging (fMRI), we made detailed measurements of the topography of human primary visual cortex. Our experiments were performed using a conventional clinical scanner, and lasted only a few minutes. We measured reliable differences in activity between cortical locations separated by less than $1.4 \mathrm{~mm}$ along continuous strips of visual cortex. The method should have broad clinical and scientific applications.

The receptive fields of neurons in human primary visual cortex follow a precisely organized retinotopic map: as a stimulus moves from the fovea to the periphery the locus of responding neurons varies from posterior to anterior portions of the calcarine sulcus, the site of human primary visual cortex ${ }^{3}$. Hence, we can use the periodic stimulus illustrated in Fig. Ia to generate waves of neural activity that travel the length of the calcarine sulcus.

As the stimulus slowly expands, each visual field location alternates between the uniform field and checkerboard. Importantly, the timecourse of the alternation depends upon visual field location; peripheral locations are delayed relative to foveal locations. When subjects view this stimulus, neural activity in the anterior portions of the calcarine should be delayed relative to activity in the posterior calcarine.
Subjects viewed four cycles of this stimulus during a 192-second session, while fMRI measurements of neural activation were continuously acquired. The fMRI signal that we measured depends upon venous blood oxygenation 4.5 . Figure $1 b$ plots the time-varying fMRI signal measured at points along the calcarine sulcus. The measured activation oscillated with the stimulus period of $48 \mathrm{~s}$, and the signal was delayed systematically along the sulcus, as expected.

To evaluate the quality of our measurements, we estimated the distance in cortex over which we could obtain reliable differences in the timecourse of the fMRI signal. To do this, we found the bestfitting (least-squares) sinusoid at the stimulus frequency for each of the four stimulus cycles at each measured cortical location. The mean of the phase-delays of these four sinusoids estimates the signal delay at that location, and the standard deviation of these phase-delays quantifies the precision of our measurements. If we define reliable separation as two standard errors, then the fMRI signal distinguished activation at points in cortex separated by $1.35 \mathrm{~mm}$, on average.

We used this paradigm to measure the cortical representation of the central 24 degrees of the visual field. We tested two subjects with a 24-degree stimulus, and analysed our data along regions of interest approximately $30 \mathrm{~mm}$ in length. We converted delay in activation to retinal eccentricity by assuming that the maximal observed delay equalled the maximal stimulus eccentricity. Figure 2 shows our results, together with estimates of the retinotopic organization of human primary visual cortex based on direct electrical stimulation ${ }^{6}, \mathrm{PET}^{2}$ and focal lesions in

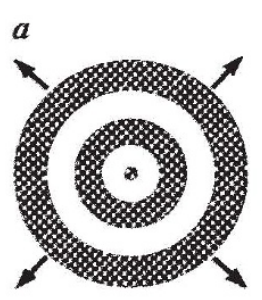

b

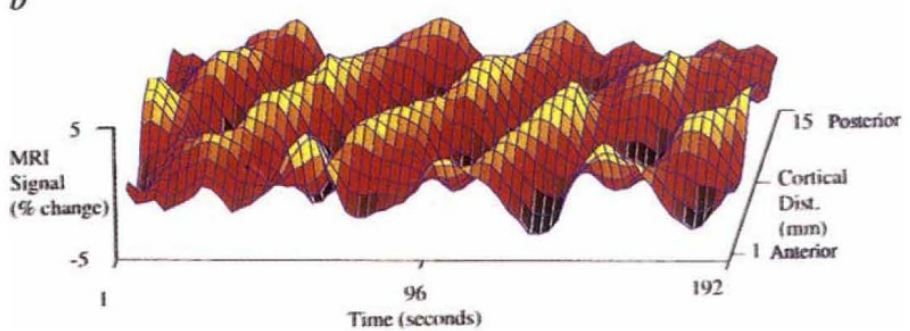

FIG. 1 Mapping receptive field locations along the calcarine sulcus. The observer viewed two concentric expanding annuli presented on a grey background. Each annulus contained a high-contrast flickering $(8.3 \mathrm{~Hz})$ radial checkerboard pattern. The outer diameter of an annulus was bounded at $10^{\circ}$; as the larger annulus disappeared, a new annulus grew in the centre of the display. The image sequence had a period of $48 \mathrm{~s}$ and was repeated four times in a single experiment. We acquired activation images every $1.5 \mathrm{~s}$ in the plane of the calcarine while subjects viewed four cycles of the stimulus over a 192-s interval. We used a clinical scanner with a $T^{*}{ }_{2}$ sensitive gradient recalled echo pulse sequence with spiral readout ${ }^{9}$. The data had an in-plane voxel size of $1.03 \mathrm{~mm}^{2}$, a through-plane resolution of $5 \mathrm{~mm}$, and were interpolated to a pixel size of $0.78 \mathrm{~mm}^{2}$ to fit a $256 \times 256$ grid. $a$, Stimulus pattern at a single moment of time. $b$, Timecourse of activation of individual pixels located along the length of the calcarine sulcus. For display purposes only, the activation data were first smoothed with a Gaussian and then every other point was plotted.

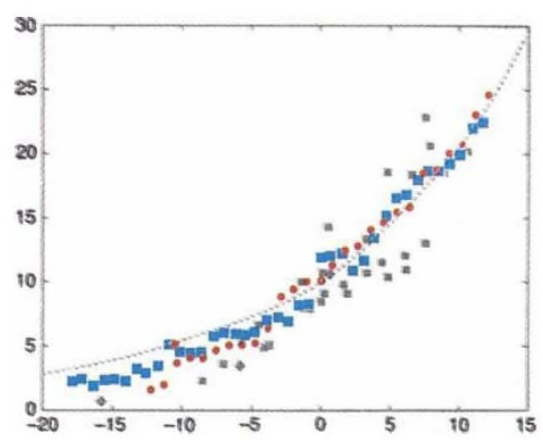

FIG. 2 Measurements of human V1 retinotopic organization. Cortical distance is measured relative to the location responding best to a stimulus at $10^{\circ}$ of retinal eccentricity. The red circles and blue squares are two subjects from the present study. Grey squares are from a microstimulation study on a single observer ${ }^{4}$; diamonds, pooled data from 5 observers in a PET study ${ }^{5}$; dashed line estimated using the locations of scotoma in stroke patients and single-cell data from non-human primates ${ }^{9}$.

stroke patients ${ }^{7}$. Note the superior density and regularity of the fMRI results, which were obtained within a brief experimental session.

The method of measuring cortical properties by temporal correlation with a periodic stimulus has wide applicability". It can be used without modification to map the retinotopic structure of other visual areas in human cortex. In addition, it should be possible to adapt our technique to measure response selectivity for properties such as colour and motion, and to measure nonvisual cortical maps. Finally, the ability to map brain areas quickly using a conventional MRI scanner should be useful clinically for diagnosis, surgical planning and studies of recovery of function.

\section{Stephen A. Engel}

David E. Rumelhart

Brian A. Wandell

Department of Psychology,

Adrian T. Lee

Gary H. Glover

Department of Radiology,

Eduardo-Jose Chichilnisky

Neuroscience Program,

Michael N. Shadien

Department of Neurobiology,

Stanford University, Stanford,

California 94305, USA

\footnotetext{
Van Essen. D. C. etal. Science 255, 419-423(1992)

2. Fox, P. T. etal. Nature 323, 806-809 (1986)

3. Holmes, G. Proc. R. Soc. B132, 348-361(1945)

4. Ogawa, S. etal. Proc. natn. Acad. Sci. U. S. A. 89. 5951- 5955 (1992).

5. Kwong, K. K. et al. Proc. natn. Acad. Sci. U.S. A. 89 5675-5679 (1992)

6. Dobelle, W. H. Turket, J, Henderson, D. C. \& Evans, J. R. Am. J. Ophthal. 88, 727-735 (1979).

7. Horton, J \& Hoyt, W. F. Arch. Ophthal. 109, 816-824 (1991)

8. Bandettini, P. A. in Proc. 1st Wkshop Functional MRI of Brain 144. 151 (Society of Magnetic Resonance in Medicine, Berkeley, 1993)

9. Meyer, C. H. et al. Mag. Reson. Med. 28. 202-213 (1992).
} 